\title{
EXTENSION OF FUNCTIONALS AND INEQUALITIES ON AN ABELIAN SEMI-GROUP'
}

\section{ROBERT KAUFMAN}

Let $(G,+)$ be an abelian semi-group and $N$ a real function on $G$ satisfying

(1) $N \geqq 0$,

(2) $N(x+y) \leqq N(x) N(y)$, for all $x, y \in G$.

The function $N^{*}(x)=\lim _{n \rightarrow \infty} N^{1 / n}(n x)$ also satisfies (1) and (2). In addition, $N^{*}(n x)=\left(N^{*}(x)\right)^{n}$.

We are interested in functions $M$ on $G$ such that

(i) $0 \leqq M \leqq N$,

(ii) $M(x+y)=M(x) M(y)$.

For such functions there is an analogue of the Hahn-Banach Theorem. Let $H \subseteq G$ be a subsemi-group of $G$ and $M$ a non-negative function on $H$ satisfying (ii) for all $x, y \in H$ and

(iii) $M(x+h) \leqq M(h) N(x)$ whenever $x \in G, h \in H$, and $h+x \in H$. Such a function admits an extension $\bar{M}$ to all of $G$ fulfilling (i) and (ii).

This extension criterion is suggested by the theorem of $\mathrm{K}$. A. Ross [1], which is concerned with complex-valued functions ("semicharacters"). In a supplement to this note the present result will be combined with the Gelfand theory of commutative Banach algebras to give an extension of Ross' theorem.

Proof. The extension $\bar{M}$ will first be constructed under this additional hypothesis:

(A) For each $x \in G$, there exists $y \in G$ such that $x+y \in H$ and $M(x+y)>0$.

It follows from (A) that $M>0$ everywhere on $H$, for if $h \in H$, $y+h \in H, M(y+h) \leqq N(y) M(h)$. Similarly, if $h_{i} \in H$ and $x+h_{1}=x+h_{2}$, $M\left(h_{1}\right)=M\left(h_{2}\right)$.

The problem can now be reduced to the case of a semi-group with cancellation. Specifically, let $R$ be the equivalence relation in $G \times G$ of pairs $(x, y)$ such that $x+z=y+z$ for some $z \in G$. Using associativity and commutativity it is easily verified that $R$ is indeed an equivalence on $G$. More is true: if we set $\pi(x)=R[x], \pi(G)$ has a unique semi-group structure such that $\pi$ is a homomorphism of $G$ onto $\pi(G)$. Also, $\pi(G)$ is a cancellation semi-group. Its elements are denoted by $\alpha, \beta, \gamma$ and $x \in \alpha$ means $\pi(x)=\alpha$.

Received by the editors July 17, 1964 and, in revised form, December 4, 1964.

1 This work was done while the author was a pre-doctoral fellow of the National Science Foundation. 
Define $L(\alpha)=\inf \{N(x) ; x \in \alpha\}$. From the usual argument for normed linear spaces it is clear that $L$ fulfills (1) and (2) in place of $N$. If $\pi\left(h_{1}\right)=\pi\left(h_{2}\right), h_{i} \in H, M\left(h_{1}\right)=M\left(h_{2}\right)$. There is thus a multiplicative functional $E$ on $\pi(H)$, defined by $E \circ \pi=M$. (A) is still true.

To complete the reduction of the problem, it is necessary to prove the analogue of (iii),

(iii)' $E(\alpha+\beta) \leqq E(\alpha) L(\beta), \quad$ whenever $\alpha \in \pi(H), \quad \beta \in \pi(G), \quad \alpha+\beta$ $\in \pi(H)$.

Let $h_{1} \in H \cap \alpha, x \in \beta . \pi\left(h_{1}+x\right) \in \pi(H)$ means the existence of $y \in G$ and $h_{2} \in H$ such that $h_{1}+x+y=h_{2}+y$. Using (A), we can replace $y$ by $h_{3} \in H: h_{1}+x+h_{3}=h_{2}+h_{3} . M\left(h_{2}\right) M\left(h_{3}\right) \leqq M\left(h_{3}\right) M\left(h_{1}\right) N(x)$ by (i) and (iii). Since $M>0$ on $H, M\left(h_{2}\right) \leqq M\left(h_{1}\right) N(x)$. Thus $E(\alpha+\beta)$ $=M\left(h_{2}\right) \leqq M\left(h_{1}\right) N(x)=E(\alpha) N(x)$. Since $x \in \beta$ was arbitrary, (iii)' holds.

If an extension, say $\bar{E}$, is possible in this reduced case, $\bar{M}=\bar{E} \circ \pi$ is a fortiori an admissible extension for the given semi-group $G$. It is more convenient to return to that $G$ and introduce the cancellation law as hypothesis for the remainder of the proof.

For $X \in G$ define $\mu=\mu(x)=\operatorname{Sup}\left\{M(y+n x+h) / N^{*}(y) M(h)\right\}^{1 / n}$, the supremum being taken over all $y, n \geqq 1, h \in H$ such that $y+n x+h$ $\in H$. It is easy to replace $N$ by $N^{*}$ in requirement (iii). Therefore

$$
M(y+n x+h) \leqq N^{*}(y+n x) M(h) \leqq N^{*}(y) N^{*}(x)^{n} M(h) .
$$

Thus $\mu \leqq N^{*}(x)$.

If $\bar{M}$ were an extension to all of $G$ satisfying (i) and (ii), necessarily $\bar{M}(x) \geqq \mu$. We shall now show that $\bar{M}(x)=\mu$ defines an extension of $M$ to the subsemi-group $H^{\prime}$ determined by $H$ and $\{x\}$ which satisfies (i)-(iii). This done, the existence of an extension to $G$ follows from Zorn's Lemma.

Let $y+n_{1} x+h_{1}=n_{2} x+h_{2}, n_{i} \geqq 0$. Claim:

(iv) $N^{*}(y) \mu^{n_{1}} M\left(h_{1}\right) \geqq \mu^{n_{2}} M\left(h_{2}\right)$.

Because $G$ has cancellation, we can take $n_{1}=0$ or $n_{2}=0$. If both are zero, (iv) follows from (iii). If $n_{1} \geqq 1, n_{2}=0$,

$$
\mu^{n_{1}} \geqq M\left(y+n_{1} x+h_{1}\right) / N^{*}(y) M\left(h_{1}\right) .
$$

This is precisely (iv). The case $n_{2}>0$ follows:

Let $y_{1}+h_{1}=n_{2} x+h_{2}$ and $y_{3}+n_{3} x+h_{3} \in H$. In (iv) we replace $\mu$ by $\left\{M\left(y_{3}+n_{3} x+h_{3}\right) / N^{*}\left(y_{3}\right) M\left(h_{3}\right)\right\}^{1 / n_{3}}$ :

$$
\begin{aligned}
N^{*}\left(y_{1}\right) M\left(h_{1}\right) & \geqq\left\{M\left(y_{3}+n_{3} x+h_{3}\right) / N^{*}\left(y_{3}\right) M\left(h_{3}\right)\right\} n_{2} / n_{3} M\left(h_{2}\right) \\
& \Leftrightarrow N^{*}\left(n_{3} y_{1}\right) N^{*}\left(n_{2} y_{3}\right) M\left(n_{3} h_{1}+n_{2} h_{3}\right) \\
& \geqq M\left(n_{2} y_{3}+n_{2} n_{3} x+n_{2} h_{3}+n_{3} h_{2}\right) .
\end{aligned}
$$


But

$$
\begin{aligned}
n_{2} h_{3}+n_{2} y_{3}+n_{2} n_{3} x+n_{3} h_{2} & =n_{2} h_{3}+n_{2} y_{3}+n_{3}\left(y_{1}+h_{1}\right) \\
& =\left(n_{2} h_{3}+n_{3} h_{1}\right)+\left(n_{3} y_{1}+n_{2} y_{3}\right) .
\end{aligned}
$$

Hence

$$
M\left(n_{3} h_{2}+n_{2} y_{3}+n_{2} n_{3} x+n_{2} h_{3}\right) \leqq M\left(n_{2} h_{3}+n_{3} h_{1}\right) N^{*}\left(n_{3} y_{1}\right) N^{*}\left(n_{2} y_{3}\right) .
$$

Since $y_{3}, n_{3}, h_{3}$ were arbitrary, we can pass to the supremum to obtain $\mu$ in (iv).

Now suppose $n_{1} x+h_{1}=n_{2} x+h_{2}, n_{i} \geqq 0$. For every positive integer $m$,

$$
\begin{aligned}
m\left(n_{1}+1\right) x+m h_{1} & =m\left(n_{2}+1\right) x+m h_{2} . \\
x+\left(m n_{1}+m-1\right) x+m h_{1} & =m\left(n_{2}+1\right) x+m h_{2} .
\end{aligned}
$$

If we apply (iv) to this, taking $x=y$, and let $m \rightarrow \infty$, we obtain $M\left(h_{1}\right) \mu^{n_{1}} \geqq M\left(h_{2}\right) \mu^{n_{2}}$. From this it follows that if we set $\bar{M}(x)=\mu$ on the subsemi-group $H^{\prime}=\{H+n x ; n \geqq 0\} \cup\{n x ; n \geqq 1\} \bar{M}$ is welldefined and multiplicative. The claim that $\bar{M}$ and $H^{\prime}$ satisfy (iii) is essentially contained in (iv).

To remove the restriction imposed by assuming (A), let $B=\{x ; y+x \in H, M(y+x)>0$, for some $y$ in $G\}$. Almost by definition, (A) holds in the subsemi-group $B$. Let $\bar{M}_{B}$ be an extension of $M$, on $H \cap B$.

The complement $C B$ is an ideal in $G$, that is, $G+C B \subseteq C B$. We define $\bar{M} \equiv 0$ on $C B$. Since $M=0$ on $H \cap C B, \bar{M}$ as defined now on all of $G$ is the desired extension.

\section{REFERENCE}

1. K. A. Ross, $A$ note on extending semicharacters to semigroups, Proc. Amer. Math. Soc. 10 (1959), 579-583.

YALE UNIVERSITY 